Pacific Journal of Mathematic 


\section{THE ASYMMETRIC PRODUCT OF THREE HOMOGENEOUS LINEAR FORMS}

\section{A. C. Woods}

Let $L_{i}=\sum_{j=1}^{3} a_{i j} x_{j}, i=1,2,3$, be three linear forms in the variables $x_{1}, x_{2}, x_{3}$ with real coefficients $a_{i j}$. A theorem of Davenport asserts that, if $\left|\operatorname{det}\left(a_{i j}\right)\right|=7$, then there exist integers $u_{1}, u_{2}, u_{3}$, not all zero, such that

$$
\left|\prod_{i=1}^{3} L_{i}\left(u_{1}, u_{2}, u_{3}\right)\right| \leqq 1 \text {. }
$$

Under the same hypothesis, W. H. Adams has asked whether, given a positive real number $u$, there exist integers $u_{1}, u_{2}, u_{3}$, not all zero, such that

$$
-u^{-1} \leqq L_{1}\left(u_{1}, u_{2}, u_{3}\right) L_{2}\left(u_{1}, u_{2}, u_{3}\right)\left|L_{3}\left(u_{1}, u_{2}, u_{3}\right)\right| \leqq u \text {. }
$$

Our objective is to prove this conjecture.

Davenport gave several proofs of his theorem [3], and other proofs have been given by Chalk and Rogers [2] and Mordell [8]. Isolation results, notably those of Davenport [6] and SwinnertonDyer [10], show that Adams conjecture is true for real $u$ in some open interval containing 1 .

The set of points $\left(L_{1}, L_{2}, L_{3}\right)$ in $R_{3}$, formed as the variables range over all integral values, is a lattice $\Lambda$ of determinant $d(\Lambda)=$ $\left|\operatorname{det}\left(a_{i j}\right)\right|$. In terms of $\Lambda$, our result is as follows.

THEOREM. If $d(\Lambda)=7$, then there exists a point $\left(x_{1}, x_{2}, x_{3}\right)$ of $\Lambda$, other than the origin, such that

$$
-u^{-1} \leqq x_{1} x_{2}\left|x_{3}\right| \leqq u,
$$

with the equality sign being necessary only if $u=1$.

The method of proof is the projective one due to Davenport [3]. We begin with three lemmas.

LeMmA 1. If $x, y, z, t$ are real numbers with $1<t^{2} \leqq 1.9$, such that the inequality

$$
-t^{2}<(n+x)(n+y)|n+z|<1
$$

is not solvable in integers $n$, then

$$
\varphi=(x-y)^{2}+(y-z)^{2}+(z-x)^{2}>14 t .
$$

We note that this is a generalization of a lemma due to 
Davenport [3].

Proof. We may assume that none of $x, y, z$ is an integer, for otherwise inequality (1) is solvable for an integer $n$. We distinguish cases according to the comparative sizes of $[x],[y],[z]$.

\section{Case 1. Two of $[x],[y],[z]$ are equal.}

As $x, y, z$ may be replaced by $x+n, y+n, z+n$ respectively, for any integer $n$, without altering either the hypothesis or the conclusion of the lemma, we may assume that two of $[x],[y],[z]$ are zero. Inequality (1) implies that

$$
|(n+x)(n+y)(n+z)|<1
$$

has no solution in integers $n$.

If $[x]=[y]=0$, then $x y(1-x)(1-y) \leqq 1 / 16$. If, further, $|x y z(x-1)(y-1)(z-1)|<1$, then (3) is solvable for one of the values $n=0,-1$. Hence, we must have $|z(z-1)| \geqq 16$, whence $z(z-1) \geqq 16$, so that either $z<-3.5$ or $z>4.5$. As $0<x, y<1$, it follows that $|x-z|>3.5$ and $|y-x|>3.5$ and therefore also $\varphi>24.5$. Thus, if $\varphi \leqq 14 t$, then $t>1.75$ and $t^{2}>1.9$, contrary to hypothesis. Hence $\varphi>14 t$.

As (3) is symmetric in $x, y, z$ the other two possibilities follow by the same argument.

Case 2. Two of $[x],[y],[z]$ differ by 1 and no two are equal.

Suppose first $[x],[y]$ differ by 1 . As we may replace $x, y, z$ by $x+n, y+n, z+n$ respectively, for any integer $n$, without altering either the hypothesis or the conclusion of the lemma, we may assume that $[x]+[y]=-1$. Again, we may replace $x, y, z$ by $-x,-y$, $-z$ respectively, without alternating the lemma, so we may assume that $z>0$. Finally, by the symmetry of $x$ and $y$ in the lemma, we may assume that $-1<x<0<y<1$.

If $z<1$ then $-1<x y z<0$, contrary to inequality (1). Therefore $z>1$. Putting $f(n)=(x+n)(y+n)(z+n)$, we have $f(1) \geqq 1$, $f(0) \leqq-t^{2}$ and $f(-1) \geqq 1$, so that $f(1)=1+e_{1}, \quad f(0)=-t^{2}-e_{2}$, $f(-1)=1+e_{3}$, where $e_{1}, \quad e_{2}, e_{3}$ are nonnegative real numbers. Introducing the new variables $\xi=x y z, \eta=x y+y z+z x$ and $\zeta=$ $x+y+z$, these equations become

$$
\begin{aligned}
& \xi+\eta+\zeta=e_{1} \\
& \xi=-t^{2}-e_{2} \\
& \xi-\eta+\zeta=2+e_{3},
\end{aligned}
$$


from which it follows that

$$
\begin{aligned}
& \zeta=1+t^{2}+\frac{1}{2} e_{1}-e_{2}+\frac{1}{2} e_{3} \\
& \eta=-1+\frac{1}{2} e_{1}-\frac{1}{2} e_{3} .
\end{aligned}
$$

Hence

$$
\begin{aligned}
\frac{1}{2} \varphi=\zeta^{2}-3 \eta & =\left(1+t^{2}+\frac{1}{2} e_{1}+e_{2}+\frac{1}{2} e_{3}\right)^{2}+3\left(1-\frac{1}{2} e_{1}+\frac{1}{2} e_{3}\right) \\
& \geqq\left(1+t^{2}\right)^{2}+3 \\
& >7 t,
\end{aligned}
$$

since the last inequality may be written in the form

$$
(t-1)\left(t^{3}+t^{2}+3 t-4\right)>0
$$

which is true as $t>1$. Thus $\phi>14 t$ as required.

We may therefore assume that $[x],[y]$ do not differ by 1 . By the symmetry of $x$ and $y$ we may suppose that $[y],[z]$ differ by 1 . As before, we may assume that $-1<z<0<y<1$. Since we are assuming that the previous cases do not arise, it follows that either $x>2$ or $x<-1$.

Suppose first that $x>2$. Then $f(1)=1+e_{1}, f(0)=-1-e_{2}$ and $f(-1)=t^{2}+e_{3}$ where $e_{1}, e_{2}, e_{3}$ are nonnegative real numbers. As before, solving these three equations for $\zeta$, $\eta$ gives

$$
\begin{aligned}
2 \varphi=(2 \zeta)^{2}-6(2 \eta) & =\left(3+t^{2}+e_{1}+2 e_{2}+e_{3}\right)^{2}+6\left(1+t^{2}-e_{1}+e_{3}\right) \\
& \geqq\left(3+t^{2}\right)^{2}+6\left(1+t^{2}\right) \\
& >28 t,
\end{aligned}
$$

since the last inequality may be written in the form

$$
(t-1)\left(t^{3}+t^{2}+13 t-15\right)>0 .
$$

Hence $\varphi>14 t$, as required.

Now suppose that $x<-1$. Then $f(1)=-t^{2}-e_{1}, f(0)=t^{2}+e_{2}$, $f(-1)=-1-e_{3}$ where $e_{1}, e_{2}, e_{3}$ are nonnegative real numbers. Proceeding as before, we obtain

$$
\begin{aligned}
2 \varphi & =\left(1+3 t^{2}+e_{1}+2 e_{2}+e_{3}\right)^{2}+6\left(1+t^{2}+e_{1}-e_{3}\right) \\
& \geqq\left(1+3 t^{2}\right)^{2}+6\left(1+t^{2}\right) \\
& >28 t,
\end{aligned}
$$

since the last inequality may be written as

$$
(t-1)\left(9 t^{3}+9 t^{2}+21 t-7\right)>0 .
$$


This completes Case 2.

The preceding two cases imply that each pair of $[x],[y],[z]$ differ by at least 2 . If each pair differ by at least 3 , then some two of $x, y, z$ differ by at least 5 , which implies that $\varphi \geqq 25>14 t$ since $t^{2} \leqq 1.9$. Therefore, we may assume from now on that some pair of $[x],[y],[z]$ differ by exactly 2 . The symmetry of $x$ and $y$ yields three cases.

Case 3. $-2<x<-1,0<y<1,2<z$.

We have $f(1) \leqq-t^{2}, f(0) \leqq-t^{2}, f(-1) \geqq 1$ and $f(-2) \geqq 1$, i.e.,

$$
\begin{aligned}
& \zeta \leqq-1-t^{2}-\eta-\xi \\
& \xi \leqq-t^{2} \\
& \zeta \geqq 2+\eta-\xi \\
& 4 \zeta \geqq 9+2 \eta-\xi .
\end{aligned}
$$

Inequalities (4) and (6) imply that

$$
\eta \leqq-\frac{1}{2}\left(t^{2}+3\right)
$$

whereas (4) and (7) yield

$$
\eta \leqq-\frac{1}{6}\left(13+4 t^{2}+3 \xi\right)
$$

Assume first that

$$
2 \eta-3 \xi \geqq 1,
$$

so that (8) and (10) give

$$
\xi \leqq-\frac{1}{3}\left(t^{2}+4\right) .
$$

By (6) and (11),

$$
\zeta \geqq \frac{1}{3}\left(t^{2}+10\right)+\eta .
$$

Now if $\eta \leqq-1 / 3\left(t^{2}+10\right)$, then

$$
\frac{1}{2} \varphi=\zeta^{2}-3 \eta \geqq t^{2}+10>11>7 t .
$$

Therefore we may assume that

$$
\eta>-\frac{1}{3}\left(t^{2}+10\right) \text {. }
$$


Then (12) and (13) imply that

$$
\begin{aligned}
\zeta^{2}-3 \eta & \geqq\left(\eta+\frac{1}{3}\left(t^{2}+10\right)\right)^{2}-3 \eta \\
& >7 t
\end{aligned}
$$

provided that the quadratic in $\eta$,

$$
\left(\eta+\frac{1}{3}\left(t^{2}+10\right)\right)^{2}-3 \eta-7 t
$$

has nonreal roots, i.e., provided that $4 t^{2}-28 t+31>0$. This inequality holds if $t<1 / 2(7-3 \sqrt{2})$, which is true since $t^{2}<1.9$. Hence we may suppose that (10) is false, i.e.,

$$
\eta<\frac{1}{2}(1+3 \xi) \text {. }
$$

We may further assume that

$$
9+2 \eta-\xi>0
$$

for otherwise, by (5),

$$
2 \eta \leqq \xi-9 \leqq-t^{2}-9<-10
$$

and therefore also

$$
\zeta^{2}-3 \eta>15>7 t
$$

Thus, by (7),

$$
\zeta^{2}-3 \eta \geqq \frac{1}{16}(9+2 \eta-\xi)^{2}-3 \eta=g(\eta), \text { say. }
$$

The quadratic $g(\eta)$ attains its minimum value at

$$
\eta=\frac{1}{2}(\xi+3)>\frac{1}{2}(1+3 \xi) \text { by }(5) \text {. }
$$

Hence, by (14),

$$
g(\eta) \geqq \frac{1}{16}(10+2 \xi)^{2}-\frac{3}{2}(1+3 \xi)=h(\xi), \text { say. }
$$

The quadratic $h(\xi)$ attains its minimum value at $\xi=4$. Suppose first that $\xi \leqq-1 / 3\left(4+t^{2}\right)$. Then

$$
g(\eta) \geqq h(\xi) \geqq \frac{1}{36}\left(11-t^{2}\right)^{2}+\frac{1}{2}\left(9+3 t^{3}\right)>7 t
$$

since 


$$
t^{4}+32 t^{2}-252 t+283>0
$$

when

$$
t^{2}<1.9
$$

Thus we may assume that

$$
\xi>-\frac{1}{3}\left(4+t^{2}\right) .
$$

As $g(\eta)$ is decreasing $\eta \leqq 1 / 2(\xi+3)$, and (15) shows that

$$
-\frac{1}{6}\left(13+4 t^{2}+3 \xi\right)<\frac{1}{2}(\xi+3),
$$

so (9) implies that

$$
g(\eta) \geqq \frac{1}{36}\left(7-2 t^{2}-3 \xi\right)^{2}+\frac{1}{2}\left(13+4 t^{2}+3 \xi\right)=j(\xi), \text { say. }
$$

But $j(\xi)$ has the minimum value $31 / 4+t^{2}$. Hence

$$
g(\eta) \geqq \frac{31}{4}+t^{2}>7 t,
$$

since $4 t^{2}-28 t+31>0$, as we have already seen. This completes the proof for Case 3 .

Case 4. $-2<x<-1,0<z<1,2<y$.

Here $f(-1) \geqq t^{2}, f(-2) \geqq t^{2}, f(1) \leqq-t^{2}, f(0) \leqq-t^{2}$ and these imply the four inequalities (4)-(7) of Case 3. Therefore the same argument applies here.

Case 5. $y<-1,0<x<1,2<z<3$.

Here $f(1) \leqq-t^{2}, f(0) \leqq-t^{2}, f(-1) \geqq 1, f(-2) \geqq 1$ which yield the four inequalities (4)-(7) of Case 3. Therefore the same argument applies here. This completes the proof of Lemma 2 .

LEMMA 2. With $g(n)=(x+n)(y+n)|z+n|$, suppose that $-t^{2}<$ $g(n)<1$ has no solution in integers $n$. If, further, $-2<z<-1<$ $x<0,1<y<2$ then $t^{2} \leqq 2$.

Proof. We have $g(2) \geqq 1, g(1) \geqq 1, g(0) \leqq-t^{2}, g(-1) \leqq-t^{2}$ and $g(-2) \geqq 1$. Now

$$
-3 g(0)+2 g(1)+g(-2) \geqq 3\left(1+t^{2}\right),
$$

i.e., 


$$
\zeta \leqq \frac{1}{2}\left(1-t^{2}\right)
$$

Also

$$
2 g(1)-g(0)+g(2) \geqq 3+t^{2}
$$

i.e.,

$$
\zeta \geqq \frac{1}{2}\left(t^{2}-3\right)
$$

Hence $1 / 2\left(t^{2}-3\right) \leqq 1 / 2\left(1-t^{2}\right)$ or $t^{2} \leqq 2$, as required.

LEMMA 3. With $g(n)$ as defined in Lemma 2, suppose that $-t^{2}<g(n)<1$ has no solution in integers $n$ when $t^{2} \geqq 1.9$. Then, with $X=x-z$ and $Y=y-z$, the point $(X, Y)$ does not lie in the plane region given by the two inequalities

$$
X Y>-2 t^{2}-\frac{1}{4}, \quad|X+Y|<\delta
$$

where $\delta=5$ if $t^{2}>2$ and $\delta=4.81$ if $1.9 \leqq t^{2} \leqq 2$.

Proof. Determine an integer $n_{0}$ such that $\left[n_{0}+z\right]=0$ and put $\lambda=n_{0}+z$, so that $0<\lambda<1$. Put $F\left(\lambda^{1}\right)=\left(X+\lambda^{1}\right)\left(Y+\lambda^{1}\right)\left|\lambda^{1}\right|$ so that the condition on $g(n)$ becomes

$$
-t^{2}<F\left(\lambda^{1}\right)<1
$$

has no solutions in real numbers $\lambda^{1} \equiv \lambda(\bmod 1)$.

Put $\zeta=X Y$ and $\eta=X+Y$ and $\lambda^{1}=\lambda, \lambda-1$ successively in (16). It follows that the point $(\zeta, \eta)$ does not lie in either of the two strips given by

$$
\frac{-t^{2}}{\lambda}<\zeta+\lambda \eta+\lambda^{2}<\frac{1}{\lambda}
$$

and

$$
\frac{-t^{2}}{1-\lambda}<\zeta+(\lambda-1) \eta+(\lambda-1)^{2}<\frac{1}{1-\lambda}
$$

Hence the point $(\zeta, \eta)$ lies in one of four regions, giving four cases, as follows.

Case a.

$$
\zeta+\lambda \eta+\lambda^{2} \leqq \frac{-t^{2}}{\lambda}
$$




$$
\zeta+(\lambda-1) \eta+(\lambda-1)^{2} \leqq \frac{-t^{2}}{1-\lambda} .
$$

Multiplying (ai) by $1-\lambda$ and (aii) by $\lambda$ and adding, we obtain

$$
\zeta \leqq-t^{2}\left(\frac{1-\lambda}{\lambda}+\frac{\lambda}{1-\lambda}\right)-\lambda+\lambda^{2} .
$$

Hence if

$$
-t^{2}\left(\frac{1-\lambda}{\lambda}+\frac{\lambda}{1-\lambda}\right)-\lambda+\lambda^{2} \leqq-2 t^{2}-\frac{1}{4}
$$

the lemma holds. But this inequality may be written in the form

$$
\left(\lambda-\frac{1}{2}\right)^{2}\left(\lambda^{2}-\lambda+4 t^{2}\right) \geqq 0,
$$

which is true since $0<\lambda<1$ and $t>1$.

Case b.

$$
\begin{gathered}
\zeta+\lambda \eta+\lambda^{2} \leqq \frac{-t^{2}}{\lambda} \\
\zeta+(\lambda-1) \eta+(\lambda-1)^{2} \geqq \frac{1}{1-\lambda} .
\end{gathered}
$$

Subtracting (bii) from (bi), we obtain

$$
\eta \leqq+\frac{1}{1-\lambda}+\frac{t^{2}}{\lambda}+2 \lambda-1
$$

Hence the lemma holds if

$$
\delta \leqq-\frac{1}{1-\lambda}-\frac{t^{2}}{\lambda}-2 \lambda+1
$$

i.e., if

(biii)

$$
2 \lambda^{3}-(3+\delta) \lambda^{2}+\left(t^{2}+\delta\right) \lambda-t^{2}<0 .
$$

In case $1.9 \leqq t^{2} \leqq 2$ and $\delta=4.81$, (biii) becomes

$$
2 \lambda^{3}-7.81 \lambda^{2}+6.71 \lambda-1.9<0,
$$

which is true for $0<\lambda<1$.

In case $t^{2}>2$ and $\delta=5$, (biii) becomes

$$
2 \lambda^{3}-8 \lambda^{2}+7 \lambda-2<0,
$$

which also holds for $0<\lambda<1$. This takes care of Case $b$.

Case c. 


$$
\begin{gathered}
\zeta+(\lambda-1) \eta+(\lambda-1)^{2} \leqq \frac{-t^{2}}{1-\lambda} \\
\zeta+\lambda \eta+\lambda^{2} \geqq \frac{1}{\lambda} .
\end{gathered}
$$

If we replace $\lambda$ by $1-\lambda$ and $\eta$ by $-\eta$ in (ci) and (cii), we obtain (bi) and (bii). Hence, by symmetry, $|\eta|>\delta$.

Case d.

$$
\begin{gathered}
\zeta+\lambda \eta+\lambda^{2} \geqq \frac{1}{\lambda} \\
\zeta+(\lambda-1) \eta+(\lambda-1)^{2} \geqq \frac{1}{1-\lambda} .
\end{gathered}
$$

Multiplying (di) by $1-\lambda$ and (dii) by $\lambda$ and adding, we obtain

$$
\zeta \geqq \frac{1-\lambda}{\lambda}+\frac{\lambda}{1-\lambda}+\lambda(\lambda-1) \geqq 1 \text {. }
$$

Hence $\zeta=X Y>0$ and $X, Y$ have the same sign. If $X, Y$ are both negative we may change them into $-X,-Y$ respectively, replace $\lambda$ by $1-\lambda$ and $\eta$ by $-\eta$ which leaves condition (16) unchanged and turns inequalities (di) and (dii) into each other. Therefore, there is no loss of generality in assuming that $X, Y$ are both positive. Again by the symmetry of $X, Y$ we may assume from now on that

$$
0<X \leqq Y
$$

If $X+\lambda \leqq Y+\lambda<2$, then one of the values $F(\lambda), F(\lambda-1)$ contradicts (16). Further, if $0<X+\lambda<1<Y+\lambda$, then $F(\lambda-1)<0$, contrary to (dii). Thus, we may assume from now on that $1<X+\lambda$ and $2<Y+\lambda$.

Assume first that $1<X+\lambda<2<Y+\lambda$. Condition (16) with $\lambda^{1}=\lambda-2$ becomes

$$
-\zeta-(\lambda-2) \eta-(\lambda-2)^{2} \geqq \frac{t^{2}}{2-\lambda}
$$

Addition of this inequality to (dii) yields

(div)

$$
\begin{aligned}
\eta & \geqq \frac{1}{1-\lambda}+\frac{t^{2}}{2-\lambda}+3-2 \lambda \\
& \geqq \frac{1}{1-\lambda}+\frac{1.9}{2-\lambda}+3-2 \lambda \\
& \geqq 4.81
\end{aligned}
$$


if $f(\lambda)=2 \lambda^{3}-4.19 \lambda^{2}+1.47 \lambda-.28 \leqq 0$. Now $f(\lambda)$ has a local maximum at $\lambda_{0}$ where $0<\lambda_{0}<1$ and

$$
f^{\prime}\left(\lambda_{0}\right)=6 \lambda_{0}^{2}-8.38 \lambda_{0}+1.47=0 .
$$

Hence $3 f\left(\lambda_{0}\right)-f^{\prime}\left(\lambda_{0}\right)=-4.19 \lambda_{0}^{2}+2.94 \lambda_{0}-.84<0$ since the discriminant is negative. Thus $f\left(\lambda_{0}\right)<0$, and as $f(0)<0$ and $f(1)<0$, it follows that $f(\lambda)<0$ and therefore also that $\eta \geqq 4.81$. Hence, if $1.9 \leqq t^{2} \leqq 2$, the lemma holds. Now assume that $t^{2}>2$. Inequality (div) implies that

$$
\begin{aligned}
\eta & \geqq \frac{1}{1-\lambda}+\frac{2}{2-\lambda}-2 \lambda+3 \\
& \geqq 5 \text { if } 2 \lambda^{3}-4 \lambda^{2}+\lambda \leqq 0,
\end{aligned}
$$

which is true if $\lambda \geqq 1-1 / \sqrt{2}$. Thus we may assume that $\lambda<$ $1-1 / \sqrt{2}$. If $2<Y+\lambda<3$, inequality (diii) may be written in the form

$$
(2-\lambda)(X+\lambda-2)(Y+\lambda-2) \leqq-t^{2},
$$

which is clearly false since $t^{2}>2$. If $3<Y+\lambda<4$ then, by Lemma $2, t^{2}>2$. Therefore we may assume that $Y+\lambda>4$. By (16) with $\lambda^{1}=\lambda-4$, it follows that

$$
-\zeta-(\lambda-4) \eta-(\lambda-4)^{2} \geqq \frac{t^{2}}{4-\lambda} .
$$

Adding this inequality to (dii), we obtain

$$
3 \eta \geqq \frac{2}{4-\lambda}+\frac{1}{1-\lambda}+15-6 \lambda \text {. }
$$

Hence

$$
\eta \geqq 5 \quad \text { if } \quad \frac{2}{4-\lambda}+\frac{1}{1-\lambda}-6 \lambda \geqq 0
$$

i.e., if

$$
-2 \lambda^{3}+10 \lambda^{2}-9 \lambda+2 \geqq 0 \text {. }
$$

The left hand side is monotone decreasing for $0 \leqq \lambda \leqq 1 / 3$ and has the value $1 / 27$ at $\lambda=1 / 3$. As $1 / 3>1-1 / \sqrt{2}$, so $\eta \geqq 5$ if $\lambda \leqq 1-$ $1 / \sqrt{2}$. Therefore, the lemma is true if $1<X+\lambda<2$, and we may assume from now on that $X+\lambda>2$.

Assume next that $2<X+\lambda<3$. In case $2<Y+\lambda<3$, condition (16) with $\lambda^{1}$ taken successively as $\lambda-2$ and $\lambda-3$ yields

$$
(2-\lambda)(X+\lambda-2)(Y+\lambda-2) \geqq 1
$$


and

$$
(3-\lambda)(X+\lambda-3)(Y+\lambda-3) \geqq 1 .
$$

Multiplying these two inequalities together and observing that

$$
-\frac{1}{4} \leqq(X+\lambda-2)(X+\lambda-3), \quad(Y+\lambda-2)(Y+\lambda-3)<0,
$$

we obtain a contradiction. Thus we may assume that $3<Y+\lambda$. Again condition (16) with $\lambda^{1}$ taken as $\lambda-2$ and $\lambda-3$ yields

$$
\zeta+(\lambda-2) \eta+(\lambda-2)^{2} \geqq \frac{1}{2-\lambda}
$$

and

$$
-\zeta-(\lambda-3) \eta-(\lambda-3)^{2} \geqq \frac{t^{2}}{3-\lambda}
$$

Adding these two inequalities together gives

$$
\eta \geqq \frac{1}{2-\lambda}+\frac{t^{2}}{3-\lambda}+5-2 \lambda \text {. }
$$

If $t^{2}>2$ then $\eta \geqq 5$ provided

$$
\frac{1}{2-\lambda}+\frac{2}{3-\lambda}-2 \lambda \geqq 0
$$

i.e.,

$$
(1-\lambda)\left(7-8 \lambda+2 \lambda^{2}\right) \geqq 0,
$$

which is true since $0<\lambda<1$. On the other hand, if $1.9 \leqq t^{2} \leqq 2$, inequality $(\mathrm{dv})$ implies $\eta \geqq 4.81$ provided

$$
\frac{1}{2-\lambda}+\frac{1.9}{3-\lambda}+5-2 \lambda \geqq 4.81
$$

i.e.,

$$
-2 \lambda^{3}+10.19 \lambda^{2}-15.85 \lambda+7.94 \geqq 0,
$$

which is true for $0<\lambda<1$, since the left hand side is monotone decreasing in this range.

We are left with the case $3<X+\lambda, Y+\lambda$. Here, if $\eta<5$, then

$$
X+Y+2 \lambda<7
$$




$$
\frac{(X+\lambda-3)+(Y+\lambda-3)}{2}<\frac{1}{2}
$$

hence, by the arithmetic-geometric mean inequality,

$$
(X+\lambda-3)(Y+\lambda-3)<\frac{1}{4}
$$

and therefore also

$$
(3-\lambda)(X+\lambda-3)(Y+\lambda-3)<\frac{3}{4}
$$

contrary to condition (16) with $\lambda^{1}=\lambda-3$. This proves Lemma 3.

Proof of the theorem. Denote by $\Lambda^{*}$ the set of points of $\Lambda$ other than 0 . We may assume that $u<1$, for otherwise, apply the transformation $T: x_{1} \rightarrow-x_{1}$ so that, if $T\left(\Lambda^{*}\right)$ has a point in the region

$$
-u \leqq x_{1} x_{2}\left|x_{3}\right| \leqq \frac{1}{u}
$$

then $\Lambda^{*}$ has a point in the region

$$
-\frac{1}{u} \leqq x_{1} x_{2}\left|x_{3}\right| \leqq u
$$

Put $\mu=\inf x_{1} x_{2}\left|x_{3}\right|$ extended over all points $\left(x_{1}, x_{2}, x_{3}\right)$ of $\Lambda$ for which $x_{1} x_{2}\left|x_{3}\right|>0$. Then, either the theorem is true, or $\mu \geqq u$. If $\mu \geqq 1$, the theorem follows immediately from Davenport's result. Hence, we may assume that $\mu<1$ and that $\Lambda^{*}$ has no point in the region given by

$$
-\frac{1}{\mu}<x_{1} x_{2}\left|x_{3}\right|<\mu .
$$

Put $\mu=\gamma^{3}$. By a classical argument, using Mahler's compactness theorem (5), there is no loss of generality in assuming that $\Lambda^{*}$ contains the point $(\gamma, \gamma, \gamma)$.

The projection of $\Lambda^{*}$ onto the plane $x_{1}+x_{2}+x_{3}=0$, parallel to the vector $(1,1,1)$ is a two-dimensional lattice, $\Lambda^{\prime}$ say, of determinant $d\left(\Lambda^{\prime}\right)=7 / \sqrt{3} \gamma$. By the classical theory of quadratic forms, there is a point of $\Lambda^{\prime}$, other than 0 , within a euclidean distance $\sqrt{14 / 3 \gamma}$ of 0 . Hence there is a point $(x, y, z)$ of $\Lambda^{*}$, linearly independent of $(\gamma, \gamma, \gamma)$, such that

$$
(x-y)^{2}+(y-z)^{2}+(z-x)^{2} \leqq \frac{14}{\gamma} .
$$


Taking $t=1 / \gamma^{3}$, if $1<t^{2} \leqq 1.9$, then by Lemma 1 , there is an integer $n$ such that

$$
-t^{2}<\left(n+\frac{x}{\gamma}\right)\left(n+\frac{y}{\gamma}\right)\left|n+\frac{z}{\gamma}\right|<1
$$

i.e.

$$
-\frac{1}{\mu}<(n \gamma+x)(n \gamma+y)|n \gamma+z|<\mu,
$$

which proves the theorem for the case when $1<t^{2} \leqq 1.9$.

If $t^{2}>1.9$, the projection of $\Lambda^{*}$ onto the plane $x_{3}=0$, parallel to the vector $(1,1,1)$, is a two-dimensional lattice $\Lambda^{\prime \prime}$ of determinant $d\left(\Lambda^{\prime \prime}\right)=7 / \gamma$. Taking $\delta=5$ if $t^{2}>2, \delta=4.81$ if $1.9<t^{2} \leqq 2$, by Minkowski's theorem on linear forms, there is a point $(X, Y, 0)$ of $\Lambda^{\prime \prime}$, other than 0 , such that

$$
|X-Y|<2 \gamma \sqrt{2 t^{2}+1 / 4}
$$

and

$$
|X+Y|<\delta \gamma
$$

since

$$
49 t^{2}<o^{2}\left(2 t^{2}+\frac{1}{4}\right)
$$

Therefore, by the arithmetic-geometric mean inequality, there is a point $(X, Y, 0)$ of $\Lambda^{\prime \prime}$, other than 0 , such that

$$
X Y>-\gamma^{2}\left(2 t^{2}+\frac{1}{4}\right)
$$

and

$$
|X+Y|<\delta \gamma .
$$

We have $X=x-z, Y=y-z$ for some point $(x, y, z)$ of $\Lambda^{*}$, linearly independent of $(\gamma, \gamma, \gamma)$. Applying Lemma 3, there is an integer $n$ such that

$$
-t^{2}<\left(n+\frac{x}{\gamma}\right)\left(n+\frac{y}{\gamma}\right)\left|n+\frac{z}{\gamma}\right|<1,
$$

i.e.,

$$
-\frac{1}{\mu}<(n \gamma+x)(n \gamma+y)|n \gamma+z|<\mu,
$$

and the theorem is proved. 


\section{REFERENCES}

1. J. W. S. Cassels, An introduction to the geometry of numbers, Springer.Verlag, Berlin, 1959.

2. J. H. H. Chalk and C. A. Rogers, On the product of three homogeneous linear forms, Proc. Camb. Phil. Soc., 47 (1951), 251-259.

3. H. Davenport, On the product of three homogeneous linear forms I, Proc. London Math. Soc., (2) 44 (1938), 412-431.

4. On the product of three homogeneous linear forms III, Proc. London Math. Soc., 45 (1939), 98-125.

5 . Note on the product of three homogeneous linear forms, J. London Math. Soc., 16 (1941), 98-101.

6. H. Davenport, On the product of three homogeneous linear forms IV, Proc. Camq. Phil. Soc., 39 (1943), 1-21.

7. K. Mahler, On lattice points in n-dimensional star bodies, I Existence theorems, Proc. Roy. Soc. London, A 187 (1946), 151-187.

8. L. J. Mordell, The product of three homogeneous linear ternary forms, J. London Math. Soc., 17 (1942), 107-115.

9. B. Segre, Lattice points in infinite domains and asymmetric diophantine approxima. tions, Duke Math. J., 12 (1945), 337-365.

10. H. P. F. Swinnerton-Dyer, On the product of three homogeneous linear forms, Act Arith., 18 (1971), 371-385.

Received August 30, 1979 and in revised form March 7, 1980.

The Ohio State University

Columbus, OH 43210 


\section{PACIFIC JOURNAL OF MATHEMATICS}

\section{EDITORS}

DONALD BABBITT (Managing Editor)

University of Galifornia

Los Angeles, California 90024

HUgo RossI

University of Utah

Salt Lake City, UT 84112

C. C. MOORE AND ANDREW OGG

University of California

Berkeley, CA 94720

\section{J. DUGUNDJI}

Department of Mathematics University of Southern California Los Angeles, California 90007

R. Finn and J. Milgram

Stanford University

Stanford, California 94305

\section{ASSOCIATE EDITORS}
R. ARENS
E. F. BECKENBACH
B. H. NeUManN
F. WOLF
K. YosHIDA

\section{SUPPORTING INSTITUTIONS}

UNIVERSITY OF ARIZONA

UNIVERSITY OF BRITISH COLUMBIA

CALIFORNIA INSTITUTE OF TECHNOLOGY

UNIVERSITY OF CALIFORNIA

MONTANA STATE UNIVERSITY

UNIVERSITY OF NEVADA, RENO

NEW MEXICO STATE UNIVERSITY

OREGON STATE UNIVERSITY
UNIVERSITY OF OREGON UNIVERSITY OF SOUTHERN CALIFONIA STANFORD UNIVERSITY UNIVERSITY OF HAWAII UNIVERSITY OF TOKYO UNIVERSITY OF UTAH WASHINGTON STATE UNIVERSITY UNIVERSITY OF WASHINGTON 


\section{Pacific Journal of Mathematics}

\section{Vol. 93, No. $1 \quad$ March, 1981}

Richard Arens, Reducing the order of a Lagrangian $\ldots \ldots \ldots \ldots \ldots \ldots \ldots$

Richard Arens, Manifestly dynamic forms in the Cartan-Hamilton treatment

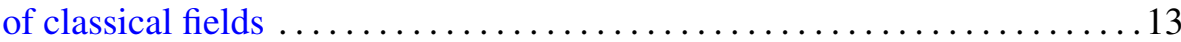

Jimmy T. Arnold, Power series rings over discrete valuation rings $\ldots \ldots \ldots 31$

Charles A. Asmuth and Joe Repka, Supercuspidal components of the

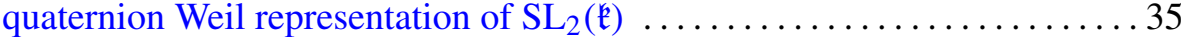

Luis A. Caffarelli and Avner Friedman, Sequential testing of several simple hypotheses for a diffusion process and the corresponding free boundary problem ................................. 49

William B. Jacob, Fans, real valuations, and hereditarily-Pythagorean fields .............................................. 95

W. J. Kim, Asymptotic properties of nonoscillatory solutions of higher order

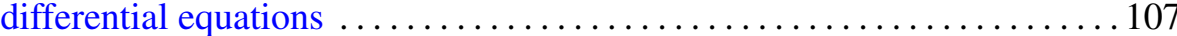

Wayne Steven Lewis, Embeddings of the pseudo-arc in $E^{2} \ldots \ldots \ldots \ldots \ldots 115$

Daniel Alan Marcus, Closed factors of normal Z-semimodules . ......... 121

Mitsuru Nakai and Leo Sario, Harmonic functionals on open Riemann surfaces ............................................. 147

John Currie Quigg, Jr., On the irreducibility of an induced

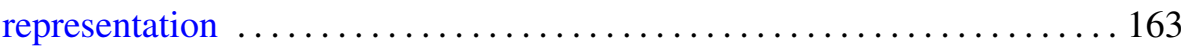

John Henry Reinoehl, Lie algebras and Hopf algebras 181

Joe Repka, Base change for tempered irreducible representations of $\mathrm{GL}(n, \mathbf{R})$

Peter John Rowley, Solubility of finite groups admitting a fixed-point-free automorphism of order $r s t$. I . . . . . . . . . . . . . . . . . . 201

Alan C. Woods, The asymmetric product of three homogeneous linear forms 